\title{
Breaking of Ensemble Equivalence in Networks
}

\author{
Tiziano Squartini, ${ }^{1,2}$ Joey de Mol, ${ }^{1,3}$ Frank den Hollander, ${ }^{3}$ and Diego Garlaschelli ${ }^{1, *}$ \\ ${ }^{1}$ Lorentz. Institute for Theoretical Physics, University of Leiden, Leiden, The Netherlands \\ ${ }^{2}$ IMT Institute for Advanced Studies, P.zza S. Ponziano 6, 55100 Lucca, Italy \\ ${ }^{3}$ Mathematical Institute, University of Leiden, Netherlands \\ (Received 5 January 2015; published 30 December 2015)
}

\begin{abstract}
It is generally believed that, in the thermodynamic limit, the microcanonical description as a function of energy coincides with the canonical description as a function of temperature. However, various examples of systems for which the microcanonical and canonical ensembles are not equivalent have been identified. A complete theory of this intriguing phenomenon is still missing. Here we show that ensemble nonequivalence can manifest itself also in random graphs with topological constraints. We find that, while graphs with a given number of links are ensemble equivalent, graphs with a given degree sequence are not. This result holds irrespective of whether the energy is nonadditive (as in unipartite graphs) or additive (as in bipartite graphs). In contrast with previous expectations, our results show that (1) physically, nonequivalence can be induced by an extensive number of local constraints, and not necessarily by longrange interactions or nonadditivity, (2) mathematically, nonequivalence is determined by a different large-deviation behavior of microcanonical and canonical probabilities for a single microstate, and not necessarily for almost all microstates. The latter criterion, which is entirely local, is not restricted to networks and holds in general.
\end{abstract}

DOI: 10.1103/PhysRevLett.115.268701

PACS numbers: 89.75.Hc, 02.10.Ox, 05.20.Gg

Background.-In statistical physics, calculating the equilibrium properties of a system with a given energy requires averaging over the so-called microcanonical ensemble $[1,2]$, i.e., the uniform distribution on the set of all particle configurations having a prescribed energy. Apart from trivial examples, this is a mathematically challenging task. Moreover, it is difficult to physically realize a situation where there is no uncertainty in the energy of the system. Therefore, it is often preferable to work with the so-called canonical ensemble [2], i.e., a probability distribution with maximal entropy on an extended set of configurations that "violate" the desired energy, but in such a way that the average energy matches the prescribed value. This is achieved through an appropriate temperature, mathematically arising as the Lagrange multiplier enforcing the prescribed average energy.

Starting with the work of Gibbs [2], the microcanonical and canonical ensembles have been shown to be equivalent in the thermodynamic limit (i.e., when the number of particles in the system tends to infinity) for physical systems with short-range interactions. The original argument is that in the canonical ensemble at fixed temperature the energy fluctuations are negligible with respect to the average energy, so that in the thermodynamic limit the canonical ensemble is effectively microcanonical with a

Published by the American Physical Society under the terms of the Creative Commons Attribution 3.0 License. Further distribution of this work must maintain attribution to the author(s) and the published article's title, journal citation, and DOI. sharp value of the energy. Today, most textbooks in statistical physics still convey the message that equivalence of ensembles holds universally for every physical system, justifying the use of energy and temperature as two different parameters giving an equivalent description.

However, in the past decades various studies have highlighted that ensemble equivalence breaks down in certain models of fluid turbulence [3,4], quantum phase separation [5-7], star formation [8,9], nuclear fragmentation [10], and networks [11-13]. Physically, it is believed that nonequivalence is associated with long-range interactions or other forms of nonadditivity [14]. However, a complete theoretical understanding of the phenomenon is still missing. Mathematically, ensemble nonequivalence has been approached in various ways $[15,16]$. In particular, the microcanonical and canonical ensembles are said to be thermodynamically equivalent [7] when the entropy and the free energy of the system are one-to-one related via a Legendre transform. The ensembles are said to be macrostate equivalent [15] when the sets of equilibrium values of the macrostate (energy, magnetization, etc.) are the same. Finally, a recent and mathematically appealing definition is that of measure equivalence [16], according to which the ensembles are said to be equivalent when the canonical probability distribution converges to the microcanonical probability distribution in the thermodynamic limit. Under certain hypotheses, the three definitions have been shown to be equivalent [16]. Moreover, large deviation theory [17] shows that the ensembles are nonequivalent on all three levels when the microcanonical specific entropy is nonconcave 
as a function of the energy density in the thermodynamic limit [16].

Here we study ensemble nonequivalence for networks with topological constraints [18-20]. Usually, ensemble nonequivalence is studied for systems in which the Boltzmann distribution describes a certain physical interaction that is encapsulated in the energy. However, as already shown by Jaynes [21], the Boltzmann distribution describes much more general ensembles of systems with given constraints, namely, all solutions to the maximumentropy problem of inference from partial information. We argue that, for any discrete enumeration problem where we need to count microcanonical configurations compatible with a given constraint, there exists a "dual" problem involving canonical configurations induced by the same constraint. We prove a general result relating measure equivalence to equivalence of the large deviation properties of microcanonical and canonical probabilities, and provide examples of networks that exhibit nonequivalence whenever the number of constraints is extensive.

Ensembles.-For $N \in \mathbb{N}$, let $\mathcal{G}_{N}$ denote the discrete set of all configurations with $N$ particles (in the examples below, all graphs with $N$ nodes). Let $\vec{C}$ denote a vector-valued function on $\mathcal{G}_{N}$. The microcanonical distribution with hard constraint $\vec{C}^{\star}$ is defined as

$$
P_{\text {mic }}(\mathbf{G})= \begin{cases}1 / \Omega_{\vec{C}^{\star}} & \text { if } \vec{C}(\mathbf{G})=\vec{C}^{\star} \\ 0 & \text { else, }\end{cases}
$$

where $\Omega_{\vec{C}^{\star}}=\left|\left\{\mathbf{G} \in \mathcal{G}_{N}: \vec{C}(\mathbf{G})=\vec{C}^{\star}\right\}\right|$ is the number of configurations that realize $\vec{C}^{\star}$. Following Jaynes [21], we introduce a dual problem involving a canonical probability distribution $P_{\text {can }}(\mathbf{G})$ defined as the solution of the maximization of the Shannon entropy $S_{N}\left(P_{\text {can }}\right)=$ $-\sum_{\mathbf{G} \in \mathcal{G}_{N}} P_{\text {can }}(\mathbf{G}) \ln P_{\text {can }}(\mathbf{G})$ subject to the soft constraint $\langle\vec{C}\rangle=\vec{C}^{\star}$, where $\langle\cdot\rangle$ denotes the average with respect to $P_{\text {can }}$, and to the normalization condition $\sum_{\mathbf{G} \in \mathcal{G}_{N}} P_{\text {can }}(\mathbf{G})=$ 1 [18]. This gives

$$
P_{\mathrm{can}}(\mathbf{G})=\frac{\exp \left[-H\left(\mathbf{G}, \vec{\theta}^{\star}\right)\right]}{Z\left(\vec{\theta}^{\star}\right)},
$$

where $H(\mathbf{G}, \vec{\theta}) \equiv \vec{\theta} \cdot \vec{C}(\mathbf{G})$ is the Hamiltonian and $Z(\vec{\theta}) \equiv$ $\sum_{\mathbf{G} \in \mathcal{G}_{N}} \exp [-H(\mathbf{G}, \vec{\theta})]$ is the partition function. Note that in Eq. (2) the parameter $\vec{\theta}$ must be set to the particular value $\vec{\theta}^{\star}$ that realizes $\langle\vec{C}\rangle=\vec{C}^{\star}[20]$. This value also maximizes the likelihood [22].

Specific relative entropy and large deviations.-The relative entropy of $P_{\text {mic }}$ with respect to $P_{\text {can }}$ is

$$
S_{N}\left(P_{\text {mic }} \| P_{\text {can }}\right)=\sum_{\mathbf{G} \in \mathcal{G}_{N}} P_{\text {mic }}(\mathbf{G}) \ln \frac{P_{\text {mic }}(\mathbf{G})}{P_{\text {can }}(\mathbf{G})} .
$$

Following Ref. [16], we say that the two ensembles are measure equivalent if their specific relative entropy is zero:

$$
s=\lim _{N \rightarrow \infty} \frac{S_{N}\left(P_{\text {mic }} \| P_{\text {can }}\right)}{N}=0 .
$$

Before considering specific cases, we make a crucial observation. Noting from the form of $H(\mathbf{G}, \vec{\theta})$ that $P_{\text {can }}\left(\mathbf{G}_{1}\right)=P_{\text {can }}\left(\mathbf{G}_{2}\right)$ when $\vec{C}\left(\mathbf{G}_{1}\right)=\vec{C}\left(\mathbf{G}_{2}\right)$ (the canonical probability is the same for all configurations with the same value of the constraint), we rewrite Eq. (3) as

$$
S_{N}\left(P_{\text {mic }} \| P_{\text {can }}\right)=\ln \frac{P_{\text {mic }}\left(\mathbf{G}^{\star}\right)}{P_{\text {can }}\left(\mathbf{G}^{\star}\right)},
$$

where $\mathbf{G}^{\star}$ is any configuration in $\mathcal{G}_{N}$ such that $\vec{C}\left(\mathbf{G}^{\star}\right)=\vec{C}^{\star}$. The equivalence condition in Eq. (4) then becomes

$$
\lim _{N \rightarrow \infty} \frac{1}{N}\left[\ln P_{\text {mic }}\left(\mathbf{G}^{\star}\right)-\ln P_{\text {can }}\left(\mathbf{G}^{\star}\right)\right]=0,
$$

which demonstrates that nonequivalence coincides with $P_{\text {mic }}\left(\mathbf{G}^{\star}\right)$ and $P_{\text {can }}\left(\mathbf{G}^{\star}\right)$ having different large deviation behavior [17]. Importantly, this condition is purely local as it involves the microcanonical and canonical probabilities of a single microstate $\mathbf{G}^{\star}$ realizing the hard constraint. This greatly simplifies previously studied global conditions involving almost all microstates [16].

Unipartite networks. - We now apply the above concepts to the class of unipartite graphs, where there is a single set of nodes among which all possible links are allowed.

Let us first consider graphs with a fixed number of links $L$; i.e., $\vec{C} \equiv L$. Writing $L=\lambda V$, where $V \equiv N(N-1) / 2$ is the number of pairs of nodes and $\lambda$ is the fraction of realized links, in the microcanonical ensemble we have

$$
\Omega_{L^{\star}}=\left(\begin{array}{c}
V \\
L^{\star}
\end{array}\right)=\left(\begin{array}{c}
V \\
\lambda^{\star} V
\end{array}\right), \quad 0<\lambda^{\star}<1 .
$$

The canonical ensemble can be obtained from Eq. (2) by setting $H(\mathbf{G}, \theta)=\theta L(\mathbf{G})$ and $p^{\star} \equiv\left(e^{-\theta^{\star}} / 1+e^{-\theta^{\star}}\right)=\lambda^{\star}$ [20]. This produces the Erdős-Rényi random graph where each pair of nodes is connected with equal probability $p^{\star}$ :

$$
P_{\text {can }}(\mathbf{G})=\left(p^{\star}\right)^{L(\mathbf{G})}\left(1-p^{\star}\right)^{V-L(\mathbf{G})} .
$$

We can now compute the relative entropy from Eq. (5) as

$$
\begin{gathered}
S\left(P_{\text {mic }} \| P_{\text {can }}\right)=-\lambda^{\star} V \ln \lambda^{\star}-\left(1-\lambda^{\star}\right) V \ln \left(1-\lambda^{\star}\right) \\
-\ln \left(\begin{array}{c}
V \\
\lambda^{\star} V
\end{array}\right)=\ln \sqrt{2 \pi \lambda^{\star}\left(1-\lambda^{\star}\right) V}+O(1 / V),
\end{gathered}
$$

where we have used Stirling's formula $n !=(n / e)^{n} \sqrt{2 \pi n}$ $[1+O(1 / n)], n \rightarrow \infty$. This gives 


$$
s=\lim _{N \rightarrow \infty} \frac{\ln \sqrt{2 \pi \lambda^{\star}\left(1-\lambda^{\star}\right) V}}{N}=0,
$$

proving ensemble equivalence. In other words, when $N \rightarrow \infty$, most graphs have a number of links that is close to the average number of links.

We next consider graphs with a fixed degree sequence, i.e., $\vec{C}=\vec{k}=\left(k_{1}, \ldots, k_{N}\right)$, where $k_{i}$ is the number of links of node $i$. This is known as the configuration model [18]. The microcanonical number $\Omega_{\vec{k}^{\star}}$ is not known in general, but asymptotic results exist in the "sparse case," where

$$
k_{\max }=\max _{1 \leq i \leq N} k_{i}=o(\sqrt{N})
$$

In this regime it is known that $[23,24]$

$$
\Omega_{\vec{k}^{\star}}=\frac{\sqrt{2}\left(\frac{2 L^{\star}}{e}\right)^{L^{\star}}}{\prod_{i=1}^{N} k_{i}^{\star} !} e^{-\left(\overline{k^{\star 2}} / 2 \overline{k^{\star}}\right)^{2}+1 / 4+o\left(N^{-1} \overline{k^{*}}\right)}
$$

where $\overline{k^{\star l}}=N^{-1} \sum_{i=1}^{N} k_{i}^{\star l}$ is the $l$-th moment of the degree distribution and $L=N \overline{k^{\star}} / 2$ is the total number of links. The canonical ensemble is described [20] by Eq. (2), where $H(\mathbf{G}, \vec{\theta})=\vec{\theta} \cdot \vec{k}(\mathbf{G})$ and $\vec{\theta}^{\star}$ is such that

$$
\sum_{j \neq i} \frac{e^{-\theta_{i}^{\star}-\theta_{j}^{\star}}}{1+e^{-\theta_{i}^{\star}-\theta_{j}^{\star}}}=k_{i}^{\star} \quad \forall i .
$$

Setting $p_{i j}^{\star} \equiv e^{-\theta_{i}^{\star}-\theta_{j}^{\star}} /\left(1+e^{-\theta_{i}^{\star}-\theta_{j}^{\star}}\right)$, we have

$$
P_{\mathrm{can}}(\mathbf{G})=\prod_{i, j}\left(p_{i j}^{\star}\right)^{g_{i j}}\left(1-p_{i j}^{\star}\right)^{1-g_{i j}},
$$

where $\prod_{i, j} \equiv \prod_{i=1}^{N} \prod_{j<i}$ and $g_{i j}$ is the entry of the adjacency matrix of G. Equation (11) ensures that $k_{\max }=o(\sqrt{L})$, a condition under which Eq. (13) is solved as [20]

$$
p_{i j}^{\star} \sim e^{-\theta_{i}^{\star}-\theta_{j}^{\star}}=\frac{k_{i}^{\star} k_{j}^{\star}}{2 L^{\star}}=o(1),
$$

where $\sim$ means that the quotient tends to 1 . This implies $\theta_{i}^{\star} \sim-\ln \left(k_{i}^{\star} / \sqrt{2 L^{\star}}\right)$ and $\ln \left(1-p_{i j}^{\star}\right) \sim-k_{i}^{\star} k_{j}^{\star} / 2 L^{\star}$. Thus,

$$
\ln P_{\operatorname{can}}\left(\mathbf{G}^{\star}\right) \sim \sum_{i=1}^{N} k_{i}^{\star} \ln k_{i}^{\star}-L^{\star} \ln \left(2 L^{\star}\right)-L^{\star} .
$$

Combining Eqs. (5), (12), and (16), we obtain

$$
\begin{aligned}
S\left(P_{\text {mic }} \| P_{\text {can }}\right) \sim & \sum_{i=1}^{N} \ln q\left(k_{i}^{\star}\right) \\
& +\left(\overline{k^{\star 2}} / 2 \overline{k^{\star}}\right)^{2}-\frac{1}{4}+o\left(N^{-1} \overline{k^{\star 3}}\right),
\end{aligned}
$$

where $q(k) \equiv k ! /(k / e)^{k} \geq \sqrt{2 \pi k}$ for $k \geq 1$. Equation (11) guarantees that the terms in the last line are $o(N)$. Denoting a limiting average over nodes with a bar, we arrive at

$$
s=\overline{\ln q\left(k^{\star}\right)} \geq \overline{\ln \sqrt{2 \pi k^{\star}}}>0,
$$

proving nonequivalence. In other words, when $N \rightarrow \infty$, most graphs in the canonical ensemble do not have a degree sequence that is close to the average degree sequence. This important result explains various recent findings, e.g., the fact that the canonical and microcanonical entropies of random regular graphs are different even in the thermodynamic limit [19] and that canonical fluctuations do not vanish in networks with local constraints [13].

As a first example we consider sparse regular networks, where every node has the same degree $k^{\star}=o(\sqrt{N})$. Then, $\overline{\ln k^{\star}}=\ln k^{\star}$, so that Eq. (18) becomes

$$
s \geq \ln \sqrt{2 \pi k^{\star}}, \quad k^{\star}=o(\sqrt{N}) .
$$

Note that when $k^{\star}$ grows with $N, s$ diverges like $\ln k^{\star}$, signaling an extreme violation of equivalence.

As a second example we consider sparse scale-free networks [25], defined by a truncated power-law degree distribution of the form $F_{N}(k) \equiv N^{-1} \sum_{i=1}^{N} 1_{\left\{k_{i}=\right.}$ $k\}=A_{\gamma} k^{-\gamma}$ with $\gamma \in(1, \infty)$ for $1 \leq k<k_{c}(N)$ and $F_{N}(k)=0$ for $k \geq k_{c}(N)$, where $\lim _{N \rightarrow \infty} k_{c}(N)=\infty$ and $k_{c}(N)=o(\sqrt{N})$. This "structural cutoff" [25] ensures Eq. (11), so that Eq. (15) is valid. Approximating $F_{N}(k)$ by a continuous distribution, we see that the normalization of $F_{N}$ implies $A_{\gamma} \approx \gamma-1$, and so Eq. (18) leads to

$$
s \geq \overline{\ln \sqrt{2 \pi k^{\star}}} \approx \frac{1}{2(\gamma-1)}+\ln \sqrt{2 \pi},
$$

confirming nonequivalence. As the tail exponent $\gamma$ decreases, the degree distribution broadens and the degree of violation of equivalence increases.

Taken together, the above examples indicate that ensemble equivalence holds when there is a single global constraint, while it is broken when there is an extensive number of local constraints. They also indicate that graphs with local constraints are always nonequivalent, irrespective of the breadth of the degree distribution.

Bipartite networks.-We now consider bipartite networks, where there are two distinct sets of nodes, and links are allowed only between the two sets. A bipartite graph $\mathbf{G}$ is specified by an $N \times M$ matrix, where $N$ and $M$ denote the numbers of nodes in the two sets. For simplicity, we constrain 
the topological properties on only one set (say, the one with $N$ nodes) and regard the other set as an "external environment." Thus, $N$ is the size of the system and the criterion in Eq. (4) still applies. For instance, we can think of our bipartite graph as a collaboration network of $N$ articles and $M$ authors. We may want to focus only on the properties of the set of articles, while regarding the "external" set of authors fixed. In particular, in the limit $N \rightarrow \infty$, we may think of $M$ as a fixed number (either finite or infinite).

If we fix only the total number $L$ of links, then the number of microcanonical configurations is still given by Eq. (7) and the canonical probability, defined via $H(\mathbf{G}, \theta)=\theta L(\mathbf{G})$, is the same as in Eq. (8), where now $V=N M$ and $p^{\star} \equiv e^{-\theta^{\star}}$ is such that $\langle L\rangle=p^{\star} V=L^{\star}$. A calculation similar to that leading to Eq. (10) shows that $s=0$, proving again ensemble equivalence.

We next fix the degree sequence $\vec{k}^{\star}=\left(k_{1}^{\star}, \ldots, k_{N}^{\star}\right)$ of the constrained set. The microcanonical configurations are enumerated exactly as $\Omega_{\vec{k}^{\star}}=\prod_{i=1}^{N}\left(\begin{array}{l}M \\ k_{i}^{\star}\end{array}\right)$. The canonical ensemble is defined by the Hamiltonian $H(\mathbf{G}, \vec{\theta})=\vec{\theta}$. $\vec{k}(\mathbf{G})$ and is still described by Eq. (14), where $\prod_{i, j}=$ $\prod_{i=1}^{N} \prod_{j=1}^{M}$ and $p_{i j}^{\star}=k_{i}^{\star} / M$ [26]. We assume that $0<$ $k_{i}^{\star}<M$ for all $i$ to avoid either disconnected nodes or fully connected nodes. A direct calculation yields

$$
s=\overline{\ln \sqrt{2 \pi k^{\star}\left(1-k^{\star} / M\right)}}
$$

(where the bar again denotes a limiting average, now over the $N$ nodes of the constrained set), proving ensemble nonequivalence. Note that here we have put no restriction on $k_{\max }$, apart from requiring $0<k_{\max }<M$. Indeed, while Eq. (18) is valid only in the sparse regime, Eq. (21) holds in the full range of connectivity.

Irrelevance of (non)additivity.-In the physics literature a connection has been conjectured between ensemble nonequivalence and nonadditivity of the energy, as induced, for instance, by long-range interactions [14]. By contrast, in our examples the only mechanism leading to nonequivalence is the presence of an extensive number of local constraints, irrespective of (non)additivity. To illustrate this result, we partition the set of $N$ nodes into two sets $\mathcal{V}_{1}$ and $\mathcal{V}_{2}$ with $N_{1}=\alpha_{1} N$ and $N_{2}=\alpha_{2} N$ nodes, respectively, where $\alpha_{1}, \alpha_{2}>0$ and $\alpha_{1}+\alpha_{2}=1$. For a given graph $\mathbf{G}$, we calculate the interaction energy between the two subsystems as $H_{\text {int }}(\mathbf{G}, \vec{\theta})=H(\mathbf{G}, \vec{\theta})-H_{1}(\mathbf{G}, \vec{\theta})-$ $H_{2}(\mathbf{G}, \vec{\theta})$, where $H_{i}(\mathbf{G}, \vec{\theta})$ denotes the restriction of $H(\mathbf{G}, \vec{\theta})$ to the set $\mathcal{V}_{i}$.

In our example of unipartite graphs with the single constraint $\vec{C}=L$, the interaction energy is $H_{\text {int }}(\mathbf{G}, \theta)=$ $\theta\left[L(\mathbf{G})-L_{1}(\mathbf{G})-L_{2}(\mathbf{G})\right]$, where $L_{i}(\mathbf{G})$ is the number of "internal" links among the nodes of $\mathcal{V}_{i}$. Thus, $H_{\text {int }}(\mathbf{G}, \theta)$ is proportional to the number of links between $\mathcal{V}_{1}$ and $\mathcal{V}_{2}$, and its expected value is

$$
\begin{aligned}
\left\langle H_{\text {int }}(\theta)\right\rangle & =\theta \frac{p}{2}\left[N(N-1)-N_{1}\left(N_{1}-1\right)-N_{2}\left(N_{2}-1\right)\right] \\
& =\theta \frac{p}{2} N^{2}\left(1-\alpha_{1}^{2}-\alpha_{2}^{2}\right)=\theta p N^{2} \alpha_{1} \alpha_{2},
\end{aligned}
$$

where $p=e^{-\theta} /\left(1+e^{-\theta}\right)$, as in Eq. (8). In the thermodynamic limit, the ratio of $\left\langle H_{\text {int }}(\theta)\right\rangle$ to the expected total energy $\langle H(\theta)\rangle=\theta p N(N-1) / 2$ is

$$
\lim _{N \rightarrow \infty} \frac{\left\langle H_{\text {int }}(\theta)\right\rangle}{\langle H(\theta)\rangle}=2 \alpha_{1} \alpha_{2}>0,
$$

which proves nonadditivity due to long-range interactions [14]. A similar result holds for the configuration model. So, unipartite networks are always nonadditive, irrespective of whether they exhibit nonequivalence.

By contrast, our examples of bipartite networks are always additive, irrespective of whether they exhibit (non) equivalence. This occurs because, when partitioning the set of $N$ nodes (e.g., articles in our previous example), both $\mathcal{V}_{1}$ and $\mathcal{V}_{2}$ remain connected only to the $M$ external nodes (e.g., authors), and not among themselves. Indeed, the Hamiltonian only couples the $N$ nodes to the external environment and we always get $H_{\text {int }}(\mathbf{G}, \theta)=0$.

Conclusion.-We found that (non)equivalence is determined by an entirely local criterion involving the largedeviation behavior of microcanonical and canonical probabilities of a single microstate rather than of almost all microstates (as generally expected [16]). This result is entirely general and is not restricted to networks. Moreover, we found that the presence of an extensive number of local constraints provides a mechanism for ensemble nonequivalence in ensembles of graphs. While in all examples known so far, nonequivalence was expected to be associated with long-range interactions or nonadditivity, in our examples (non)equivalence is only determined by the number of constraints, irrespective of (non)additivity. From a practical point of view, graphs with local constraints are routinely used as null models to detect empirical patterns or to reconstruct networks from partial information $[13,18-20,26]$. So far, choosing between microcanonical and canonical implementations [13] of these null models has been perceived as a mere matter of convenience. However, our findings imply that one should make a careful and principled choice, as results obtained using different ensembles may differ substantially. The same considerations might extend to other ensembles of systems with many constraints, applications of which range from biology (e.g., conformational ensembles) to finance and neuroscience (e.g., time series ensembles).

T. S. is supported by the EU project SIMPOL (Grant No. 610704). F.d.H. is supported by ERC Advanced Grant No. 267356-VARIS and NWO Gravitation Grant No. 024.002.003-NETWORKS. D. G. is supported by the EU project MULTIPLEX (Contract No. 317532) and the Dutch Econophysics Foundation (Stichting Econophysics, Leiden). 
* Corresponding author. garlaschelli@lorentz.leidenuniv.nl

[1] L. Boltzmann, Wiener Berichte 76, 373 (1877).

[2] J. W. Gibbs, Elementary Principles of Statistical Mechanics (Yale University Press, New Haven, CT, 1902).

[3] R. S. Ellis, K. Haven, and B. Turkington, J. Stat. Phys. 101, 999 (2000).

[4] R. S. Ellis, K. Haven, and B. Turkington, Nonlinearity 15, 239 (2002).

[5] M. Blume, V. J. Emery, and R. B. Griffiths, Phys. Rev. A 4, 1071 (1971).

[6] J. Barré, D. Mukamel, and S. Ruffo, Phys. Rev. Lett. 87, 030601 (2001).

[7] R. S. Ellis, H. Touchette, and B. Turkington, Physica (Amsterdam) 335A, 518 (2004).

[8] D. Lynden-Bell, Physica (Amsterdam) 263A, 293 (1999).

[9] P.-H. Chavanis, Astron. Astrophys. 401, 15 (2003).

[10] M. D’Agostino et al., Phys. Lett. B 473, 219 (2000).

[11] J. Barré and B. Goncalves, Physica (Amsterdam) 386A, 212 (2007).

[12] C. Radin and L. Sadun, J. Phys. A 46, 305002 (2013).

[13] T. Squartini, R. Mastrandrea, and D. Garlaschelli, New J. Phys. 17, 023052 (2015).
[14] A. Campa, T. Dauxois, and S. Ruffo, Phys. Rep. 480, 57 (2009).

[15] H. Touchette, R. S. Ellis, and B. Turkington, Physica (Amsterdam) 340A, 138 (2004).

[16] H. Touchette, J. Stat. Phys. 159, 987 (2015).

[17] F. den Hollander, Large Deviations, in Fields Institute Monographs Vol. 14 (American Mathematical Society, Providence RI, 2000).

[18] J. Park and M. E. J. Newman, Phys. Rev. E 70, 066117 (2004).

[19] K. Anand and G. Bianconi, Phys. Rev. E 80, 045102 (2009).

[20] T. Squartini and D. Garlaschelli, New J. Phys. 13, 083001 (2011).

[21] E. T. Jaynes, Phys. Rev. 106, 620 (1957).

[22] D. Garlaschelli and M. I. Loffredo, Phys. Rev. E 78, 015101 (R) (2008).

[23] E. A. Bender, Discrete Math. 10, 217 (1974).

[24] B. D. McKay and N. C. Wormald, Combinatorica 11, 369 (1991).

[25] M. Boguñá, R. Pastor-Satorras, and A. Vespignani, Eur. Phys. J. B 38, 205 (2004).

[26] F. Saracco, R. Di Clemente, A. Gabrielli, and T. Squartini, Sci. Rep. 5, 10595 (2015). 